Corrigendum

\title{
Corrigendum to "Preservation of Myocardial Perfusion and Function by Keeping Hypertrophied Heart Empty and Beating for Valve Surgery: An In Vivo MR Study of Pig Hearts"
}

\author{
Jian Wang, ${ }^{1,2,3}$ Bo Xiang, ${ }^{2,4}$ Jixian Deng, ${ }^{2,3}$ Hung-Yu Lin, ${ }^{2}$ Darren H. Freed, ${ }^{2,3,5}$ \\ Rakesh C. Arora, ${ }^{2,3,6}$ and Ganghong Tian ${ }^{2,3}$ \\ ${ }^{1}$ Department of Vascular Surgery, Union Hospital, Tongji Medical College, Huazhong University of Science and Technology, \\ Wuhan 430022, China \\ ${ }^{2}$ National Research Council of Canada, 435 Ellice Avenue, Winnipeg, MB, Canada R3B $1 Y 6$ \\ ${ }^{3}$ Department of Physiology, Faculty of Medicine, University of Manitoba, 727 McDermot Avenue, Winnipeg, MB, Canada R3E $3 P 5$ \\ ${ }^{4}$ Department of Pharmacology and Therapeutics, Faculty of Medicine, University of Manitoba, 753 McDermot Avenue, \\ Winnipeg, MB, Canada R3E 0T6 \\ ${ }^{5}$ Division of Cardiac Surgery, University of Alberta Hospital, 8440-112 Street, Edmonton, AB, Canada T6G $2 B 7$ \\ ${ }^{6}$ Cardiac Science Program, Institute of Cardiovascular Science, St. Boniface General Hospital, 409 Tache Avenue, \\ Winnipeg, MB, Canada R2H $2 A 6$ \\ Correspondence should be addressed to Jian Wang; jianwang1030@126.com
}

Received 25 December 2017; Accepted 28 December 2017; Published 31 December 2017

Copyright ( 2017 Jian Wang et al. This is an open access article distributed under the Creative Commons Attribution License, which permits unrestricted use, distribution, and reproduction in any medium, provided the original work is properly cited.

In the article titled "Preservation of Myocardial Perfusion and Function by Keeping Hypertrophied Heart Empty and Beating for Valve Surgery: An In Vivo MR Study of Pig Hearts" [1], affiliation number one was given incorrectly. The corrected affiliation is shown above.

\section{References}

[1] J. Wang, B. Xiang, J. Deng et al., "Preservation of myocardial perfusion and function by keeping hypertrophied heart empty and beating for valve surgery: an in vivo MR study of pig hearts," BioMed Research International, vol. 2017, Article ID 4107587, 11 pages, 2017. 


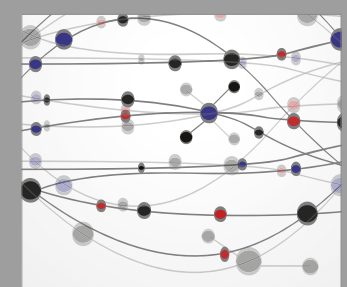

The Scientific World Journal
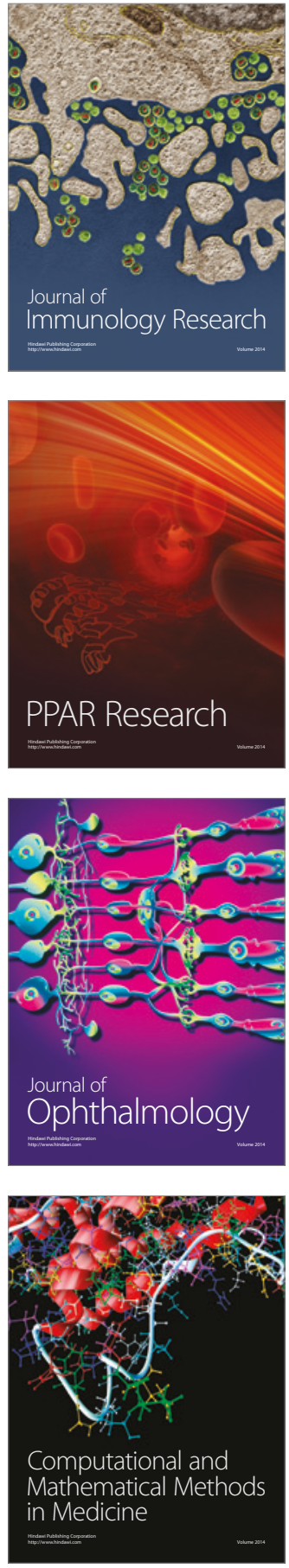

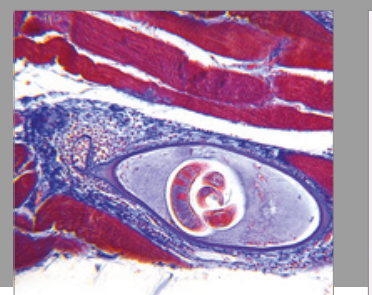

Gastroenterology Research and Practice
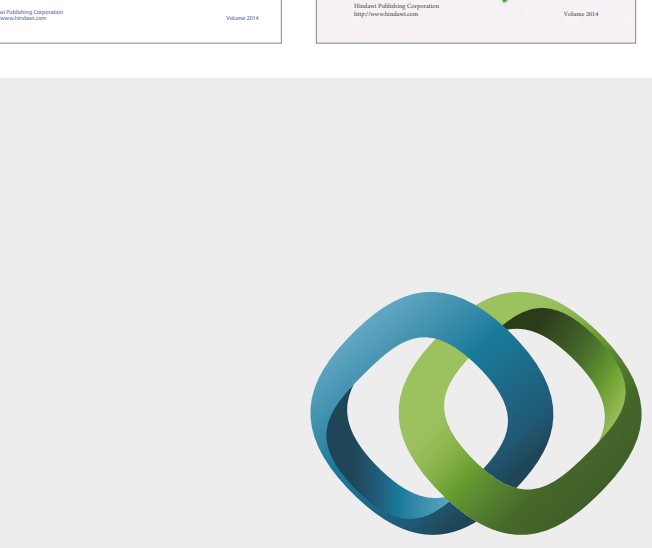

\section{Hindawi}

Submit your manuscripts at

https://www.hindawi.com
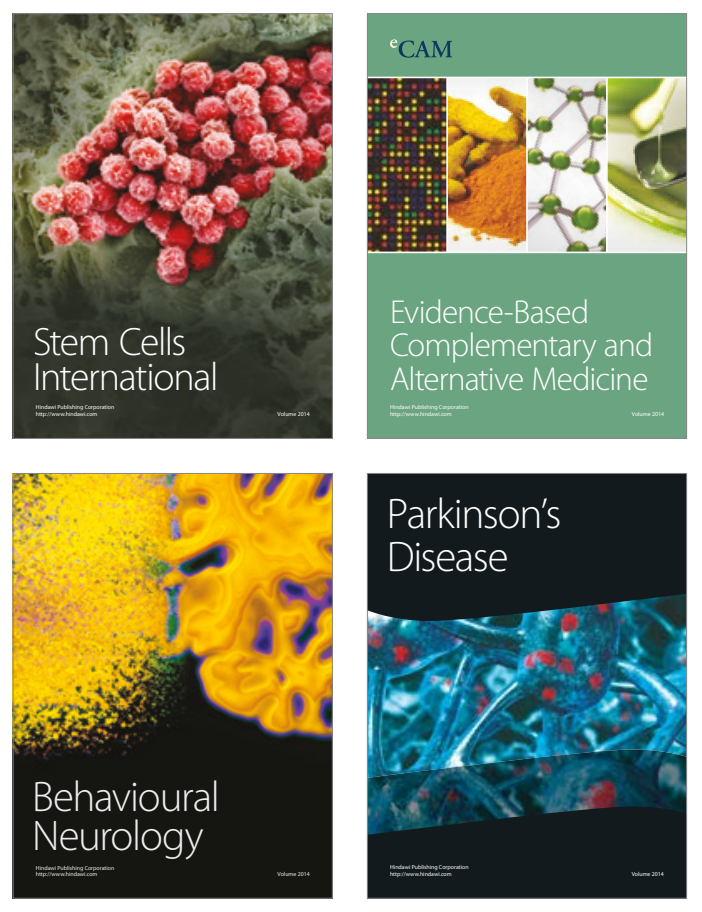
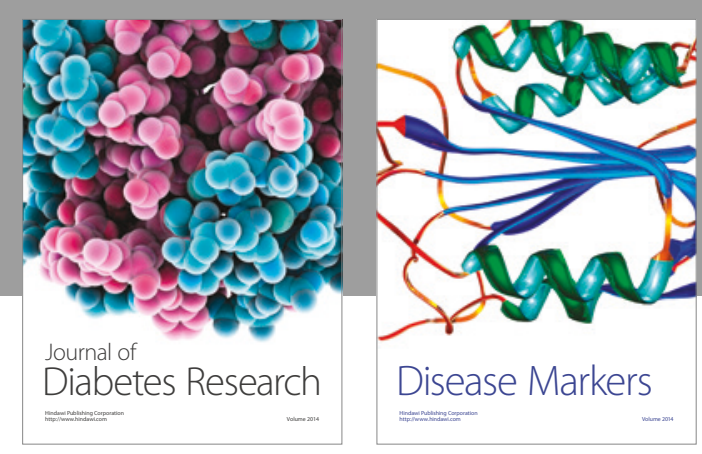

Disease Markers
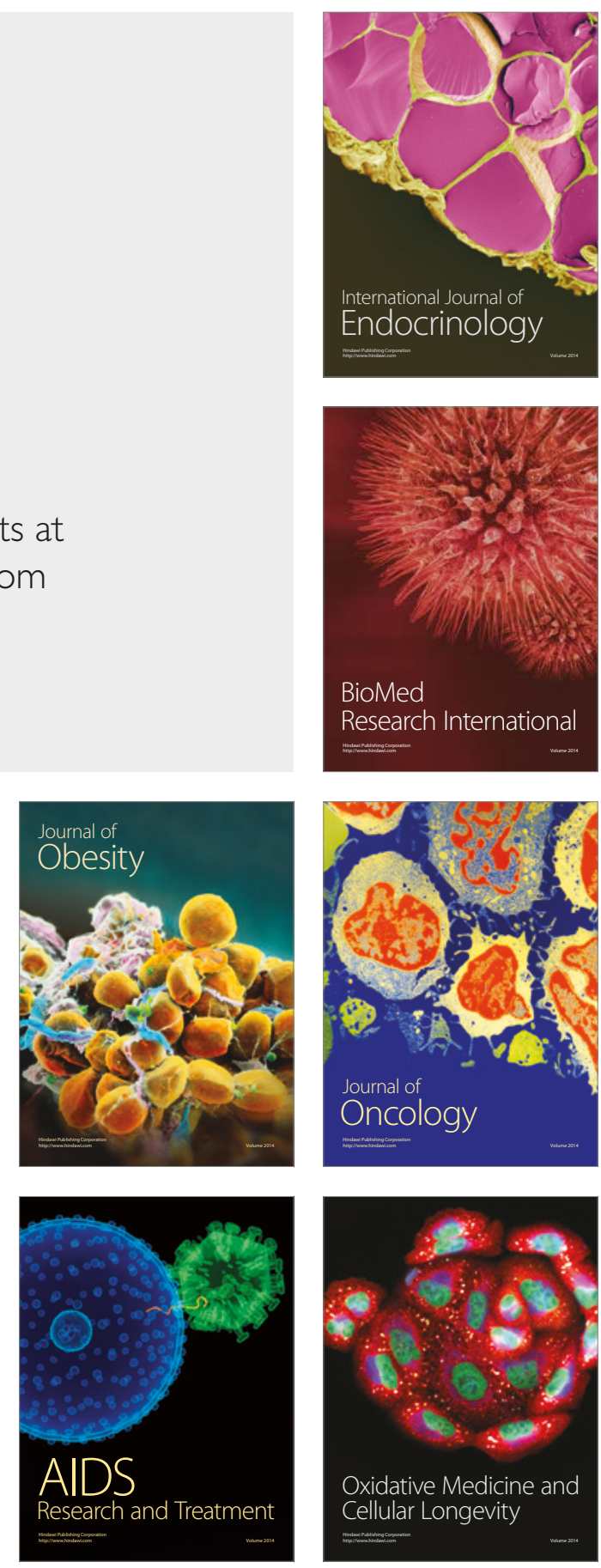